\title{
In-plane Anisotropic Raman Spectroscopy of Van der Waals $\alpha-\mathrm{MoO}_{3}$
}

Muqian Wen ${ }^{\prime \#}$, Xuexian Chen ${ }^{*}$, Zebo, Zheng ${ }^{\star}$, Shaozhi Deng ${ }^{\star}$, Zhibing Li', Weiliang Wang $^{{ }^{*}}$, and Huanjun Chen *

† State Key Laboratory of Optoelectronic Materials and Technologies, Guangdong

Province Key Laboratory of Display Material and Technology, School of Physics,

Sun Yat-sen University, Guangzhou 510275, China

+ State Key Laboratory of Optoelectronic Materials and Technologies, Guangdong

Province Key Laboratory of Display Material and Technology, School of Electronics and Information Technology, Sun Yat-sen University, Guangzhou 510275, China

The formula for calculating polycrystalline Raman intensity is:

$$
\begin{gathered}
I_{S}=V \frac{\omega_{s}^{4}}{c^{4}} \frac{\hbar}{2 \omega_{j}} \frac{e^{\hbar \omega_{j} / k_{B} T}}{e^{\hbar \omega_{j} / k_{B} T}-1} \int_{0}^{2 \pi} \int_{0}^{\pi} \int_{0}^{2 \pi} \mid \vec{e}_{s} \cdot r^{T}(\psi, \theta, \varphi) \cdot R_{j} \cdot r(\psi, \theta, \varphi) \\
\left.\cdot \vec{e}_{l}\right|^{2} \sin \theta d \psi d \theta d \varphi
\end{gathered}
$$

Where $r(\psi, \theta, \varphi)$ is a series of rotation operation:

$$
r(\psi, \theta, \varphi)=\left[\begin{array}{ccc}
1 & 0 & 0 \\
0 & \cos \varphi & -\sin \varphi \\
0 & \sin \varphi & \cos \varphi
\end{array}\right]\left[\begin{array}{ccc}
\cos \theta & -\sin \theta & 0 \\
\sin \theta & \cos \theta & 0 \\
0 & 0 & 1
\end{array}\right]\left[\begin{array}{ccc}
1 & 0 & 0 \\
0 & \cos \psi & -\sin \psi \\
0 & \sin \psi & \cos \psi
\end{array}\right]
$$




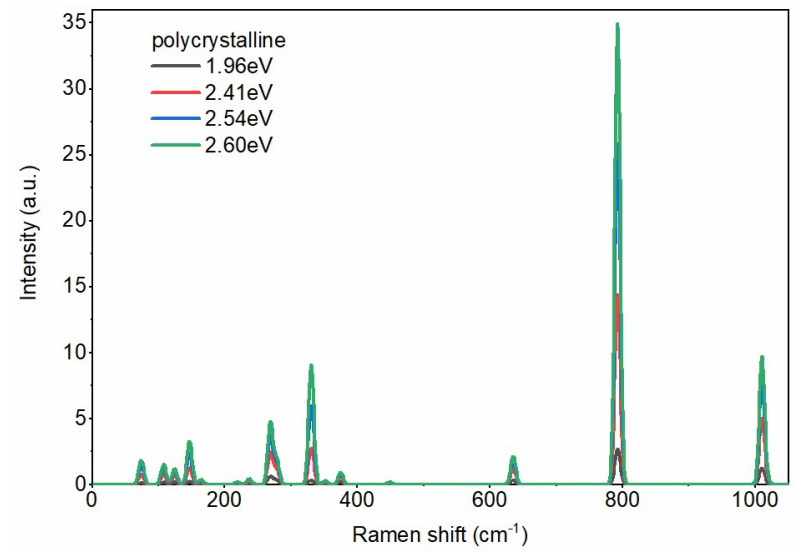

Figure S1 Theoretical Raman spectra of polycrystalline $\alpha-\mathrm{MoO}_{3}$ in backscattering configuration with laser photon energy $1.96 \mathrm{eV}$ (magenta), $2.41 \mathrm{eV}$ (red), $2.54 \mathrm{eV}$ (blue) and $2.60 \mathrm{eV}$ (green), respectively. The Raman shift lines are broadened with Gaussian function with width of $4 \mathrm{~cm}^{-1}$.

\section{A}

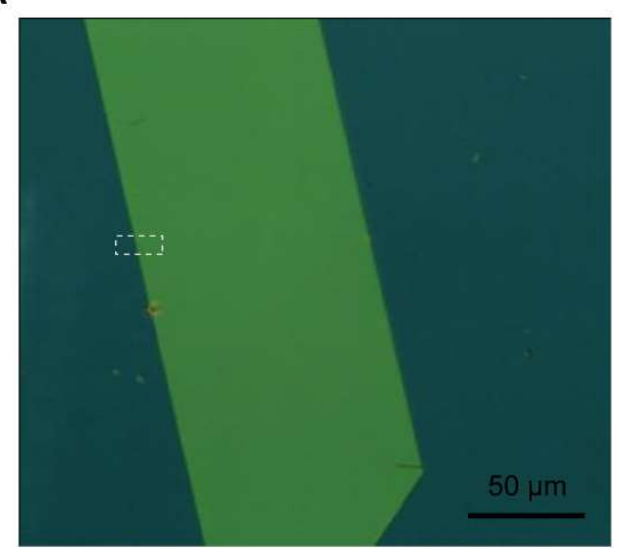

B
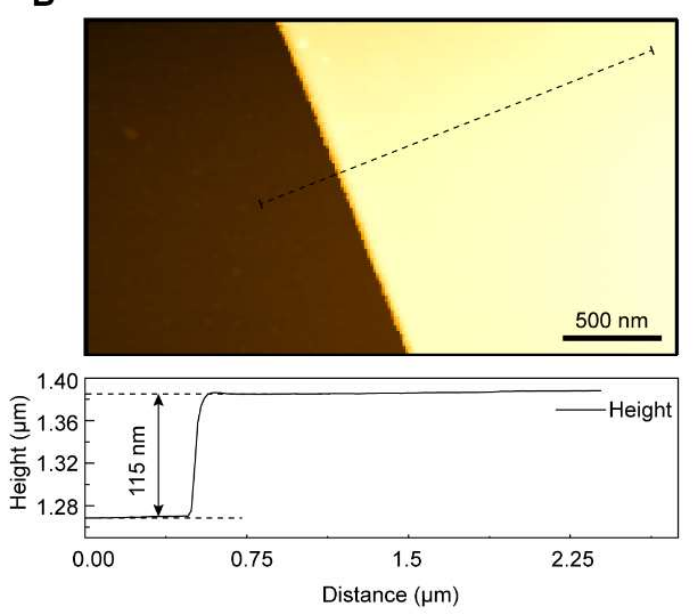

Figure S2 A) Optical microscope image of the $\alpha-\mathrm{MoO}_{3}$ flake used in our study. B) AFM topology of the sample corresponding to (A). Line profile indicates the thickness of the flake. 